OPEN ACCESS

Edited by:

Luigi Daniele Notarangelo, Laboratory of Clinical Immunology and Microbiology (NIAID), United States

Reviewed by:

William Horsnell,

University of Cape Town, South Africa lan Marriott,

University of North Carolina at Charlotte, United States

*Correspondence:

Parisa Kalantar parisa.kalantari@tufts.edu

Specialty section:

This article was submitted to

Microbial Immunology,

a section of the journa

Frontiers in Immunology

Received: 18 September 2018 Accepted: 08 January 2019

Published: 30 January 2019

Citation:

Kalantari P, Bunnell SC and Stadecker MJ (2019) The C-type Lectin Receptor-Driven, Th17 Cell-Mediated Severe Pathology in Schistosomiasis: Not All Immune Responses to Helminth Parasites Are Th2 Dominated.

Front. Immunol. 10:26. doi: 10.3389/fimmu.2019.00026

\section{The C-type Lectin Receptor-Driven, Th17 Cell-Mediated Severe Pathology in Schistosomiasis: Not All Immune Responses to Helminth Parasites Are Th2 Dominated}

\author{
Parisa Kalantari *, Stephen C. Bunnell and Miguel J. Stadecker \\ Department of Immunology, Tufts University School of Medicine, Boston, MA, United States
}

Schistosomiasis is a major helminthic disease in which damage to the affected organs is orchestrated by a pathogenic host CD4 T helper (Th) cell-mediated immune response against parasite eggs. In the case of the species Schistosoma mansoni, the resulting granulomatous inflammation and fibrosis takes place in the liver and intestines. The magnitude of disease varies greatly from individual to individual but in a minority of patients, there is severe disease and death. S. mansoni infection in a murine model similarly results in marked strain variation of immunopathology. In the most commonly examined mouse strain, C57BL/6 (BL/6), there is relatively mild hepatic pathology arising in a Th2-dominated cytokine environment. In contrast, CBA mice develop decisively more severe lesions largely driven by proinflammatory IL-17-producing Th17 cells. Dendritic cells (DCs) from CBA mice differ sharply with those from BL/6 mice in that they vastly over-express the C-type lectin receptor (CLR) CD209a (SIGNR5), a homolog of human DC-SIGN, which senses glycans such as those produced by schistosome eggs. Silencing of CD209a, and recent studies with CD209a KO CBA mice have shown that this receptor is crucial to induce the pathogenic Th17 cell response; indeed, CD209a KO mice display markedly reduced immunopathology akin to that seen in BL/6 mice. Mechanistically, CD209a synergizes with the related CLRs Dectin-2 and Mincle to stimulate increased DC production of IL-1 $\beta$ and IL-23, necessary for pathogenic Th17 cell development. These findings denote key molecular underpinnings of disease variability based on selection and function of contrasting Th cell subsets.

Keywords: C-type lectin receptor, CD209a, DC-SIGN, Schistosoma mansoni, Th17 cells, immunopathology

\section{SCHISTOSOME HELMINTHS, HUMAN SCHISTOSOMIASIS, AND THE EXPERIMENTAL MURINE MODEL}

Schistosomes represent a genus of trematode helminths (blood flukes) that have parasitized mammals for millions of years before parasite and host acquired present-day configurations. Infection in humans occurs when free swimming parasite larvae (cercariae), released from fresh water vector snails, penetrate the skin. Over several weeks, the parasites evolve through several stages to finally home in specific venous beds, where adults mate and lay eggs of which a fraction is 
trapped in regional organs. Infection with the species Schistosoma mansoni (S. mansoni) targets the liver and intestines, where the eggs trigger granulomatous inflammation and fibrosis. There is great variation in the severity of the resulting hepatic immunopathology. Most often there is relatively limited symptomatology and good survival; however, a minority of patients develop severe disease leading to portal hypertension, splenomegaly, ascites, gastro-intestinal hemorrhage, and death (1).

Today, schistosomiasis is considered to be the second most important human parasitic disease and remains a major health and socio-economic concern in many parts of the developing world (2). Despite important measures in sanitation, vector control and treatment programs, new cases are on the rise. Expectations to produce a protective vaccine against the parasite have so far not materialized and the specter of parasite resistance to drug therapy looms large. Although substantial progress has been made in elucidating the immune nature of the schistosome egg-induced inflammation, there is still much to be learned about the mechanisms underlying the marked variation in severity of disease, which is the main focus of the present article.

Our laboratory has investigated the immune response and immunopathology in murine infection with $S$. mansoni using a well-established experimental model that bears remarkable resemblance to the human condition, in terms of immunopathogenesis, anatomic localization, and heterogeneity of disease severity among various mouse strains.

\section{THE SPECTRUM OF HOST IMMUNE RESPONSE AND IMMUNOPATHOLOGY ELICITED BY SCHISTOSOME EGGS}

All stages of the schistosome life cycle inside the mammalian host elicit immune responses, but only the eggs are the target of a vigorous granulomatous inflammatory reaction that is the cause of the observed morbidity. The hepatic inflammation is mediated by CD4 Th cells specific for egg antigens, making schistosomiasis an immunologically mediated disease, since the damage to the affected tissues is largely inflicted by the host's own immune system rather than by the parasite itself. However, despite identical infection protocols, there is considerable lesional variation among different inbred mouse strains (3). CBA, C3H, SJL, and MOLF mice all develop large egg granulomas with inflammatory cell spillage into the surrounding hepatic parenchyma, whereas in BL/6 mice, the most commonly used strain in experimental schistosomiasis research, they are more confined and significantly smaller in size (4-7). Additionally, the high-pathology strains display significantly enhanced hepatomegaly, splenomegaly as well as mesenteric lymphadenomegaly. Crosses between the high-pathology strains with BL/6 yield low-pathology F1 offspring, and in F2 cohorts the pathology typically ranges from low to severe (6). Different high- $\mathrm{x}$ low-pathology crosses reveal considerable variation in the number and location of the quantitative trait loci involved in the control of immunopathology, indicating that the molecular mechanisms mediating these responses are complex (6). In our studies, we have largely focused on $\mathrm{CBA}$ and BL/6 mice as our model high- vs. low-pathology strains.

Cytokines produced by egg antigen-stimulated liver, mesenteric lymph node or spleen $\mathrm{T}$ cell populations from infected mice are faithful correlates of immunopathology. In BL/6 mice, an initial short-lived elevation in IFN- $\gamma$-producing Th1 cells gives way to a largely host-protective Th2 (IL-4, IL-5, IL-13)-dominated environment (8), whereas high-pathology strains sustain a proinflammatory Th1 and Th17 (IL-17) cell-polarized response alongside the Th2 response (6). The Th1/Th17 phenotype is largely driven by antigen presenting cells (APCs) expressing markers consistent with classical activation, while the Th2 response is supported by alternatively activated APCs (9). A prompt and effective down-modulation of the proinflammatory response is critical to limit immunopathology and enhance host survival (10).

In vitro lymphoid cell cultures have traditionally been stimulated with a soluble preparation of schistosome egg antigens (SEA) (11). However, in our lab we have gradually replaced SEA with freshly harvested live eggs, as they obviate the risk of losing temperature-sensitive, short-lived or short-ranged components, and more physiologically embody those released during the in vivo infection. A vast array of egg-secreted products, mostly glycoproteins, have been identified (12), with some of the most abundant ones having been the subject of closer analysis. IPSE (SmEP-25) (13), Omega 1 (14), Kappa 5 (15), largely tested in BL/6 mice, stimulate Th2 cytokine production, whereas Sm-p40 elicits a prominent oligoclonal Th1/Th17 cell response in the CBA strain $(4,6,16,17)$.

\section{Th17 CELLS ARE DRIVERS OF SEVERE PATHOLOGY IN SCHISTOSOMIASIS}

In the early 2000's the newly discovered Th17 cells, rather than Th1 cells, were found to play a central role in mediating the immunopathology in a number of autoimmune conditions, including experimental allergic encephalomyelitis, collageninduced arthritis, psoriasis and colitis (18). Th17 cells are a distinct lineage of proinflammatory CD4 T cells characterized by expressing the master lineage-specific transcription factor ROR $\gamma t$ and secreting the key signature cytokine IL-17A, herein termed IL-17 $(19,20)$. Analogous to the autoimmune diseases, we demonstrated that Th17 cells, rather than the originally presumed Th1 cells, are the main drivers of pathology in schistosome-infected CBA mice (21). Th17 cells were also shown to drive high pathology in a surrogate model afforded by simultaneously immunizing infected BL/6 mice with SEA emulsified in complete Freund's adjuvant (CFA) $(9,22)$. Neither SEA or CFA by themselves were able to achieve disease exacerbation and a Th17 cell response, suggesting that this effect was due to concurrent stimulation of relevant innate receptors with egg antigens plus mycobacterial antigens contained in CFA (23), analogous to what has also been described with the use of other microbial products (24). Since the description of their role in the development of severe immunopathology in S. mansoni 
infection, Th17 cells were also reported to mediate hepatic egginduced pathology in murine infection with S. japonicum (2527 ), and to be associated in humans with bladder pathology caused by eggs of $S$. hematobium (28). Th17 responses have also been associated with Ascaris suum infection, as has morbidity in filariasis (29-32).

Among the splenic APC populations purified from eggstimulated CBA mice, DCs optimally activated Th17 cell responses in in vitro co-cultures with $\mathrm{T}$ cells, while macrophages, $B$ cells and neutrophils were ineffective in this regard (33). GM-CSF-elicited bone marrow-derived (BMDCs) from CBA mice were also effective inducers of IL-17, whereas BL/6-derived BMDCs induced little to no IL-17 production (33). Analysis of supernatants from live egg-stimulated CBA BMDCs revealed that IL- $1 \beta$ and IL-23 were the critical cytokines inducing Th17 cell differentiation and that they cooperate to achieve maximal IL-17 production by $\mathrm{T}$ cells $(6,21,34)$.

The secretion of mature IL- $1 \beta$ requires the transcriptional activation of the IL- $1 \beta$ locus and the proteolytic processing of cytoplasmic pro-IL-1 $\beta$. The latter event is controlled by a family of intracellular immune receptors that are assembled into inflammasomes, which facilitate the activation of caspase1 , enable the cleavage of pro-IL-1 $\beta$, and trigger the release of bioactive IL-1 $\beta(35,36)$. A previous relevant study described the participation of the NLRP3 inflammasome in the secretion of IL-1 $\beta$ following stimulation with SEA and a TLR agonist, with neither stimulus being capable of doing so independently (37). This contrasts with our model in which live eggs can deliver all of the signals necessary for IL- $1 \beta$ production by DCs, although the precise identity of these compounds is currently unknown.

\section{CD209a EXPRESSION ON BMDCs ENHANCES THE PRODUCTION OF PATHOGENIC Th17-ASSOCIATED PROINFLAMMATORY CYTOKINES}

A genome-wide gene profiling analysis to account for genetic differences between $\mathrm{CBA}$ and BL/6 BMDCs surprisingly disclosed a significant disparity in the expression of several Ctype lectin receptors (CLRs). CLRs are a family of calciumdependent pattern recognition receptors bearing conserved carbohydrate-recognition domains that bind a wide variety of host- and pathogen-derived glycans (38). Specifically, expression of CD209a (SIGNR5, mDC-SIGN), a murine homolog of human DC-specific ICAM-3 grabbing non-integrin (DCSIGN/CD209), was strikingly higher $(\sim 18 \mathrm{x})$ in unstimulated BMDCs from CBA mice relative to BL/6 mice (33). Similar changes in CD209a expression were observed in the spleens and liver egg granulomas of infected CBA vs. BL/6 mice by mRNA analysis, flow cytometry, and immunofluorescent tissue staining (33).

To test whether the enhanced expression of CD209a on BMDCs from CBA mice plays a crucial role in the development of egg-induced Th17 responses and severe immunopathology we used lentivirally-expressed short hairpin RNAs (shRNAs) to suppress CD209a in CBA BMDCs. This treatment markedly decreased the production of IL-1 $\beta$ and IL-23 following stimulation with live eggs, and profoundly attenuated IL-17 production by co-cultured $\mathrm{CD} 4 \mathrm{~T}$ cells. Conversely, the lentiviral over-expression of CD209a conferred on egg-stimulated BL/6 BMDCs the ability to produce IL- $1 \beta$ and IL-23, and to induce Th17 cells $(33,39)$. Thus, the relative inability of BL/6 mice to mount pathogenic Th17 responses in response to schistosome eggs cannot be attributed to intrinsic defects present in $\mathrm{BL} / 6 \mathrm{~T}$ cells, and is instead linked to the inability of BL/6 BMDCs to elicit these responses.

To facilitate analogous studies in vivo, we produced CD209a $\mathrm{KO}$ mice backcrossed to the CBA background. After a 7week infection, these mice were far less prone to hepatic granulomatous inflammation, splenomegaly and mesenteric lymphadenomegaly than their wild-type CBA counterparts, and instead resembled wild-type BL/6 mice. Immune cells from the spleen and livers of the CD209a-deficient CBA mice, as opposed to wild-type CBA mice, produced less IL-1 $\beta$, IL23, IL-17, IFN $\gamma$, IL-6, and TNF $\alpha$, and more of the Th2associated cytokines IL-4, IL-5, and IL-13. The egg-specific recall responses of CD209a KO spleen, MLN and liver granuloma cells yielded similar CD209a-dependent shifts in cytokine production. In vitro, CD209a KO CBA BMDCs failed to produce significant IL-1 $\beta$ and IL-23 in response to live eggs, and could not induce effective IL-17 production by CD4 T cells, even though CD209a did not affect the levels of TNF- $\alpha$. Thus, both in vivo and in vitro, CD209a selectively enhances proinflammatory pathways associated with the induction of Th17 responses (40).

\section{THE CD209/DC-SIGN FAMILY OF CLRs AND THEIR EGG LIGANDS}

Although the functions of CD209a have not been well characterized, human DC-SIGN has been intensively studied given its roles in adhesion, pathogen uptake, and pathogendependent signaling. Like most members of this family of CLRs, DC-SIGN is a type II transmembrane protein, with a cytoplasmic tail, a transmembrane region, a tetrameric neck domain, and an extracellular C-type lectin domain. DC-SIGN, present on monocyte-derived DCs and immature tissue DCs, recognizes and internalizes a wide range of mannose and fucose-containing glycans derived from fungi, bacteria, viruses, parasites, and, of relevance to this work, schistosomes (41-43). In addition, DCSIGN shapes the induction of adaptive cellular and humoral immune responses, favoring Th1 responses when presented with mannosylated ligands, and favoring Th2 or follicular $\mathrm{T}$ helper cell $\left(\mathrm{T}_{\mathrm{FH}}\right)$ responses when presented with fucosylated ligands (44-46). DC-SIGNR (CD209L), a closely related human paralog, interacts with similar pathogens, but is primarily found on endothelial cells and macrophages and favors mannosylated glycans $(41,47,48)$.

The murine receptors within this family are less well understood, as they are not direct orthologs of the human receptors. Of seven paralogous CD209-related proteins, only 
three clearly function as membrane-bound receptors: CD209a (SIGNR5), CD209b (SIGNR1), and CD209d (SIGNR3) $(41,49)$. CD209a and CD209d are expressed in DCs, and may function in a manner analogous to DC-SIGN, while CD209b is expressed in a pattern resembling that of DC-SIGNR. The murine CD209 proteins also bind a spectrum of mannose- and fucose-containing ligands (50).

Schistosome eggs express the relevant mannosylated and fucosylated glycans, which are largely attached to glycoproteins. Typical motifs include Lewis $\mathrm{X}\left(\mathrm{Le}^{\mathrm{x}}\right)$, Poly $\mathrm{Le}^{\mathrm{x}}$, pseudo Lewis $\mathrm{Y}\left(\mathrm{Le}^{\mathrm{y}}\right)$, LacdiNac (LDN), and variously fucosylated (F) forms of LDN (F-LDN, LDN-F, F-LDN-F) (51-54). Many of these glycan moieties bind human DC-SIGN, suggesting that murine homologs of DC-SIGN function as receptors for schistosome egg glycans [reviewed in (54)]. Indeed, CD209b interacts with multiple $S$. mansoni antigens in vitro; however, the loss of CD209b has no impact on schistosome-induced immunopathology (55).

Because no ligands of CD209a have been discovered, we examined whether the sugar-binding site in the Ctype lectin domain is required for the biological activity of CD209a. Mutations affecting the calcium-dependent fucose- or mannose-binding site prevented the expression of CD209a. However, nearby surfaces involving residues analogous to Y164 and L202 of CD209a influence the specificity of CLR-glycan interactions $(56,57)$. When we replaced two nearby and distinctive surface-exposed residues with residues common to DC-SIGN, DC-SIGNR, and CD209d (CD209a R211S/D212G, Figure 1) the resulting receptor was surface-expressed but could not enhance the production of proinflammatory cytokines by BL/6-derived BMDCs

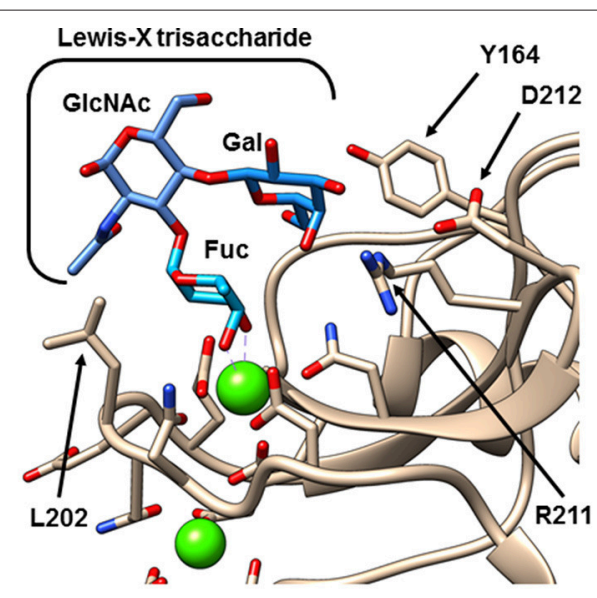

CD209a Predicted LeX Binding (DC-SIGNR 1s|6)

FIGURE 1 | Hypothetical model of CD209a binding Lewis X. A model of murine CD209a was based on an existing DC-SIGNR structure (PDB 1sl6) using the Phyre2 web interface. The Lewis X trisaccharide (blue) is predicted to bind CD209a (tan). Side chains involved in calcium or sugar binding are shown. Calcium ions are shown in green; side chain oxygen and nitrogen atoms are shown in red and blue, respectively. Residues analogous to Y164 and L202 influence ligand selectivity in DC-SIGN and DC-SIGNR. In this model of CD209a, R211, and D212 are oriented toward the ligand-binding site. challenged with live schistosome eggs. This strongly suggests that the specific recognition of a schistosome-derived ligand by CD209a is required to initiate proinflammatory signaling pathways (40).

To clarify the nature of the schistosome-derived ligand(s) that triggers CD209a-dependent signals we generated recombinant CD209a chimeras via a tandem affinity purification tag placed at the amino-terminus of the full-length receptor. Using this recombinant protein, we were able to screen a glycan library for the first time. Although no overwhelming candidates were identified, there was a trend toward the recognition of multi-antennary glycan structures containing Lewis-related antigens, consistent with the properties of the DC-SIGN receptor family.

\section{CD209a ACTIVATES Raf-1 TO PROMOTE INFLAMMATION}

DC-SIGN and CD209a are capable of enhancing the production of proinflammatory Th1- and Th17-associated cytokines following pathogen challenge. The relevant mechanisms are best understood for human DC-SIGN, which signals via a constitutively associated adaptor, lymphocyte-specific protein 1 (LSP1). LSP1 provides a platform for the recruitment of a tripartite "signalosome" consisting of KSR1, CNK, and Raf-1. When mannosylated ligands are delivered concurrently with LPS, this LSP1-associated "signalosome" mediates the activation of Ras and Raf-1. In turn, Raf-1 phosphorylates the canonical NF- $\kappa$ B subunit p65 (RelA) on S276. This directs the acetylation of p65, which enhances the activity of p65 and enables p65 to antagonize non-canonical RelB signaling $(44,58)$. Although less is known about the mechanisms by which the murine CD209 family members signal, we determined that CD209a-dependent increases in the production of IL-1 $\beta$ and IL-23 following stimulation with live eggs require the activity of Raf-1 (40) (Figure 2). This effect is specific, as perturbations of Raf-1 did not block the production of egg-induced TNF $\alpha$. Thus, the schistosome egg-induced responses mediated by CD209a may require LSP1-dependent pathways analogous to those employed by human DC-SIGN during the recognition of mannosylated ligands. Although CD209a enhances Th17 responses and promotes immunopathology in murine schistosomiasis, DCSIGN can also favor the Th2 responses when challenged by fucosylated ligands. To date, the relevant mechanisms have only been studied in human cells, where fucosylated ligands drive the dissociation of the KSR1/CNK/Raf-1 signalosome from LSP1, precluding the activation of Raf-1 (44). Instead, an alternative LSP1-dependent signaling complex is assembled, resulting in the nuclear translocation of $\mathrm{Bcl}-3$, which pairs with p50/p50 homodimers to drive the transcription of Th2associated cytokines and chemokines (45). It remains to be seen whether the murine homologs of DC-SIGN are capable of directing Th2-promoting events when presented with fucosylated ligands, or whether the murine CD209 family members have evolved fixed, specialized, proinflammatory functions. 


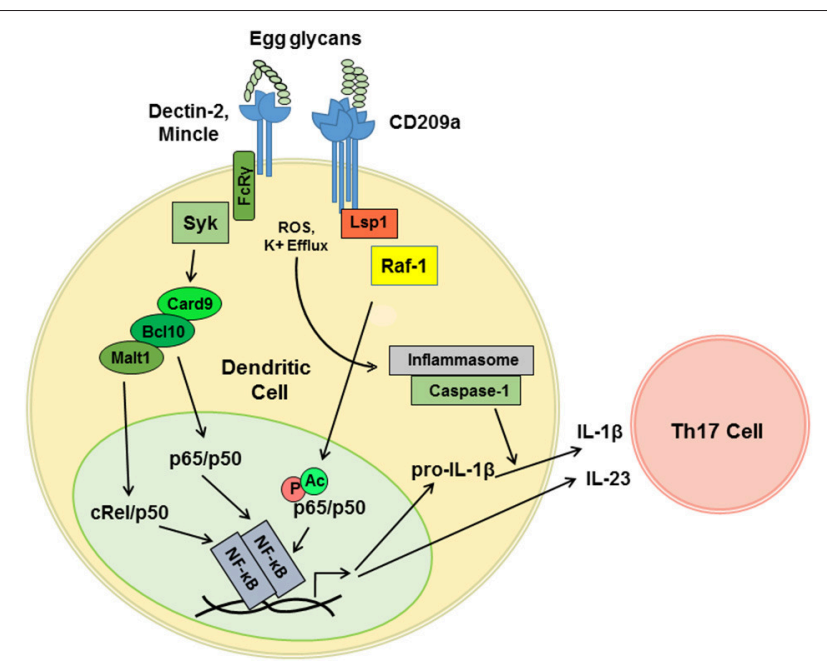

FIGURE 2 | CD209a synergizes with Dectin-2-related receptors to drive the pro-inflammatory cytokine responses triggered by live schistosome eggs. Schistosome egg ligands interact with Dectin-2, Mincle, and CD209a. Dectin-2 and Mincle initiate immunoreceptor-like signals that are mediated by the FcR $\gamma$ subunit and the tyrosine kinase Syk. Downstream of Syk, Card9 nucleates complexes containing Bcl10 and Malt1, which drive the translocation of the canonical NF-kB heterodimers required for the transcription of proinflammatory cytokine mRNAs. CD209a ligation activates Raf-1 most likely via the scaffold LSP1. By analogy with human DC-SIGN, Raf- 1 is predicted to drive the phosphorylation $(P)$ and acetylation $(A c)$ of p65, and to thereby enhance and sustain the activation of NF-kB. Schistosome eggs also activate the NLRP3 inflammasome, likely via $\mathrm{ROS}$ and $\mathrm{K}^{+}$efflux, which enables the processing of pro-IL-1 $\beta$ and secretion of mature IL-1 $\beta$. IL-1 $\beta$ together with IL-23 induce the differentiation and activation of Th17 cells.

\section{CD209a DRIVES INFLAMMATION IN SYNERGY WITH DECTIN-2 AND MINCLE}

Our recent work suggests that variable function of DC-SIGN may not need to be dictated by the recognition of distinct ligands but may instead be determined by the combinatorial detection of ligands by additional receptors. In particular, we confirmed that the pattern-recognition receptor Dectin-2 plays a role in the detection of schistosome-derived glycans and revealed that synergy between CD209a and Dectin-2 maximizes the proinflammatory cytokine responses initiated by live schistosome eggs $(37,40)$. Dectin-2 is a member of a group of CLRs that includes Mincle, Dectin-3, and the dendritic cell activating receptors, Dcar1 and Dcar2 (59). These receptors respond to "non-self" glycans and signal via the gamma subunit of the high affinity IgE receptor (Fcerlg, known as FcR $\gamma$ ), which

\section{REFERENCES}

1. Colley DG, Bustinduy AL, Secor WE, King CH. Human schistosomiasis. Lancet (2014) 383:2253-64. doi: 10.1016/S0140-6736(13) 61949-2

2. Hotez PJ, Fenwick A. Schistosomiasis in Africa: an emerging tragedy in our new global health decade. PLoS Negl Trop Dis. (2009) 3:e485. doi: 10.1371/journal.pntd.0000485 enables antigen receptor-like signaling via the tyrosine kinase Syk $(60,61)$. Using egg-challenged BL/6-derived knockout BMDCs expressing CD209a, we determined that Mincle, FcR $\gamma$, and Syk also contribute to the induction of proinflammatory signals (40).

In agreement with prior reports revealing a role for the Card9/Bcl10/Malt1 complex downstream of Dectin-2 (62), we demonstrated that the loss of Card9 or the inhibition of Malt1 hinder the production of IL- $1 \beta$ and IL- 23 by DCs stimulated with live eggs (40). This is consistent with the role of Malt1 in the Dectin-2-mediated activation of the NF- $\mathrm{KB}$ subunit cRel, which is a key player in the induction of Th17 responses (63). Therefore, we postulate that a LSP1-dependent, Raf-1activating pathway initiated by CD209a synergizes with a Card9and Malt1-dependent pathway initiated by Dectin-2, and, further, that this synergy is mediated via the prolongation of canonical $\mathrm{NF}-\kappa \mathrm{B}$ signaling and the suppression of the non-canonical and Bcl-3-dependent NF- $\mathrm{BB}$ complexes associated with Th2-biased immune responses (Figure 2).

\section{CONCLUSIONS}

Infection with schistosomes can result in clinical syndromes of dissimilar severity. In the experimental model, with genetically inbred mouse strains, identical parasite loads and similar infection protocols, disease heterogeneity is largely based on the predominant activation of pro- vs. anti-inflammatory Th cell subsets. Here we have described the synergistic crosstalk of egg antigen-sensing CLRs CD209a with Dectin-2 and Mincle, and analyzed how the interaction of their respective signaling pathways results in the activation of pathogenic Th17 cell responses and the consequent development of severe egg-induced immunopathology. The broader implication of our findings is the knowledge that receptors can collaborate with one another to shape immunopathological responses in a combinatorial fashion and that their signaling pathways can be individually targeted for the purpose of ameliorating disease.

\section{AUTHOR CONTRIBUTIONS}

All authors listed have made a substantial, direct and intellectual contribution to the work, and approved it for publication.

\section{FUNDING}

This work was supported by National Institute of Allergy and Infectious Disease grant R01 AI018919 to MJS. 
to eggs of Schistosoma mansoni. Eur J Immunol. (1997) 27:666-70. doi: 10.1002/eji.1830270314

5. Smith PM, Jacque B, Conner JR, Poltorak A, Stadecker MJ. IRAK-2 regulates IL-1-mediated pathogenic Th17 cell development in helminthic infection. PLoS Pathog. (2011) 7:e1002272. doi: 10.1371/journal.ppat.1002272

6. Larkin BM, Smith PM, Ponichtera HE, Shainheit MG, Rutitzky LI, Stadecker MJ. Induction and regulation of pathogenic Th17 cell responses in schistosomiasis. Semin Immunopathol. (2012) 34:873-88. doi: 10.1007/s00281-012-0341-9

7. Smith PM, Shainheit MG, Bazzone LE, Rutitzky LI, Poltorak A, Stadecker MJ. Genetic control of severe egg-induced immunopathology and IL-17 production in murine schistosomiasis. J Immunol. (2009) 183:3317-23. doi: 10.4049/jimmunol.0901504

8. Pearce EJ, Caspar P, Grzych JM, Lewis FA, Sher A. Downregulation of Th1 cytokine production accompanies induction of Th2 responses by a parasitic helminth, Schistosoma mansoni. J Exp Med. (1991) 173:159-66. doi: $10.1084 /$ jem.173.1.159

9. Rutitzky LI, Stadecker MJ. Exacerbated egg-induced immunopathology in murine Schistosoma mansoni infection is primarily mediated by IL17 and restrained by IFN-gamma. Eur J Immunol. (2011) 41:2677-87. doi: 10.1002/eji.201041327

10. Herbert DR, Holscher C, Mohrs M, Arendse B, Schwegmann A, Radwanska $\mathrm{M}$, et al. Alternative macrophage activation is essential for survival during schistosomiasis and downmodulates $\mathrm{T}$ helper 1 responses and immunopathology. Immunity (2004) 20:623-35. doi: 10.1016/S1074-7613(04)00107-4

11. Boros DL, Warren KS. Delayed hypersensitivity-type granuloma formation and dermal reaction induced and elicited by a soluble factor isolated from Schistosoma mansoni eggs. J Exp Med. (1970) 132:488-507. doi: $10.1084 /$ jem.132.3.488

12. Cass CL, Johnson JR, Califf LL, Xu T, Hernandez HJ, Stadecker MJ, et al. Proteomic analysis of Schistosoma mansoni egg secretions. Mol Biochem Parasitol. (2007) 155:84-93. doi: 10.1016/j.molbiopara.2007.06.002

13. Schramm G, Gronow A, Knobloch J, Wippersteg V, Grevelding CG, Galle J, et al. IPSE/alpha-1: a major immunogenic component secreted from Schistosoma mansoni eggs. Mol Biochem Parasitol. (2006) 147:9-19. doi: 10.1016/j.molbiopara.2006.01.003

14. Everts B, Perona-Wright G, Smits $\mathrm{HH}$, Hokke $\mathrm{CH}$, van der Ham AJ, Fitzsimmons CM, et al. Omega-1, a glycoprotein secreted by Schistosoma mansoni eggs, drives Th2 responses. J Exp Med. (2009) 206:1673-80. doi: $10.1084 /$ jem. 20082460

15. Schramm G, Hamilton JV, Balog CI, Wuhrer M, Gronow A, Beckmann S, et al. Molecular characterisation of kappa-5, a major antigenic glycoprotein from Schistosoma mansoni eggs. Mol Biochem Parasitol. (2009) 166:4-14. doi: 10.1016/j.molbiopara.2009.02.003

16. Finger E, Brodeur PH, Hernandez HJ, Stadecker MJ. Expansion of CD4 T cells expressing a highly restricted TCR structure specific for a single parasite epitope correlates with high pathology in murine schistosomiasis. Eur $J$ Immunol. (2005) 35:2659-69. doi: 10.1002/eji.200526344

17. Hernandez HJ, Edson CM, Harn DA, Ianelli CJ, Stadecker MJ. Schistosoma mansoni: genetic restriction and cytokine profile of the $\mathrm{CD} 4+\mathrm{T}$ helper cell response to dominant epitope peptide of major egg antigen Sm-p40. Exp Parasitol. (1998) 90:122-30. doi: 10.1006/expr.1998.4309

18. Iwakura $\mathrm{Y}$, Nakae S, Saijo S, Ishigame H. The roles of IL-17A in inflammatory immune responses and host defense against pathogens. Immunol Rev. (2008) 226:57-79. doi: 10.1111/j.1600-065X.2008.00699.x

19. McKenzie BS, Kastelein RA, Cua DJ. Understanding the IL-23-IL-17 immune pathway. Trends Immunol. (2006) 27:17-23. doi: 10.1016/j.it.2005.10.003

20. Bettelli E, Oukka M, Kuchroo VK. T(H)-17 cells in the circle of immunity and autoimmunity. Nat Immunol. (2007) 8:345-50. doi: 10.1038/ni0407-345

21. Shainheit MG, Smith PM, Bazzone LE, Wang AC, Rutitzky LI, Stadecker MJ. Dendritic cell IL-23 and IL-1 production in response to schistosome eggs induces Th17 cells in a mouse strain prone to severe immunopathology. J Immunol. (2008) 181:8559-67. doi: 10.4049/jimmunol.181.12.8559

22. Rutitzky LI, Lopes da Rosa JR, Stadecker MJ. Severe CD4 T cell-mediated immunopathology in murine schistosomiasis is dependent on IL-12p40 and correlates with high levels of IL-17. J Immunol. (2005) 175:3920-6. doi: 10.4049/jimmunol.175.6.3920
23. Rutitzky LI, Hernandez HJ, Stadecker MJ. Th1-polarizing immunization with egg antigens correlates with severe exacerbation of immunopathology and death in schistosome infection. Proc Natl Acad Sci USA. (2001) 98:13243-8. doi: $10.1073 /$ pnas. 231258498

24. Perona-Wright G, Lundie RJ, Jenkins SJ, Webb LM, Grencis RK, MacDonald AS. Concurrent bacterial stimulation alters the function of helminth-activated dendritic cells, resulting in IL-17 induction. J Immunol. (2012) 188:2350-8. doi: 10.4049/jimmunol.1101642

25. Zhang Y, Chen L, Gao W, Hou X, Gu Y, Gui L, et al. IL-17 neutralization significantly ameliorates hepatic granulomatous inflammation and liver damage in Schistosoma japonicum infected mice. Eur J Immunol. (2012) 42:1523-35. doi: 10.1002/eji.201141933

26. Chen D, Luo X, Xie H, Gao Z, Fang H, Huang J. Characteristics of IL17 induction by Schistosoma japonicum infection in C57BL/6 mouse liver. Immunology (2013) 139:523-32. doi: 10.1111/imm.12105

27. Wang B, Liang S, Wang Y, Zhu XQ, Gong W, Zhang HQ, et al. Th17 down-regulation is involved in reduced progression of schistosomiasis fibrosis in ICOSL KO mice. PLoS Negl Trop Dis. (2015) 9:e0003434. doi: 10.1371/journal.pntd.0003434

28. Mbow M, Larkin BM, Meurs L, Wammes LJ, de Jong SE, Labuda LA, et al. Thelper 17 cells are associated with pathology in human schistosomiasis. J Infect Dis. (2013) 207:186-95. doi: 10.1093/infdis/jis654

29. Anuradha R, George PJ, Chandrasekaran V, Kumaran PP, Nutman TB, Babu S. Interleukin 1 (IL-1)- and IL-23-mediated expansion of filarial antigen-specific Th17 and Th22 cells in filarial lymphedema. Clin Vaccine Immunol. (2014) 21:960-5. doi: 10.1128/CVI.00257-14

30. Babu S, Bhat SQ, Pavan Kumar N, Lipira AB, Kumar S, Karthik C, et al. Filarial lymphedema is characterized by antigen-specific Th1 and th17 proinflammatory responses and a lack of regulatory $\mathrm{T}$ cells. PLoS Negl Trop Dis. (2009) 3:e420. doi: 10.1371/journal.pntd. 0000420

31. Katawa G, Layland LE, Debrah AY, von Horn C, Batsa L, Kwarteng A, et al. Hyperreactive onchocerciasis is characterized by a combination of Th17-Th2 immune responses and reduced regulatory T cells. PLoS Negl Trop Dis. (2015) 9:e3414. doi: 10.1371/journal.pntd.0003414

32. Nogueira DS, Gazzinelli-Guimaraes PH, Barbosa FS, Resende NM, Silva CC, de Oliveira LM, et al. Multiple exposures to ascaris suum induce tissue injury and mixed Th2/Th17 immune response in mice. PLoS Negl Trop Dis. (2016) 10:e0004382. doi: 10.1371/journal.pntd.0004382

33. Ponichtera HE, Shainheit MG, Liu BC, Raychowdhury R, Larkin BM, Russo JM, et al. CD209a expression on dendritic cells is critical for the development of pathogenic Th17 cell responses in murine schistosomiasis. J Immunol. (2014) 192:4655-65. doi: 10.4049/jimmunol.1400121

34. Shainheit MG, Lasocki KW, Finger E, Larkin BM, Smith PM, Sharpe AH, et al. The pathogenic Th17 cell response to major schistosome egg antigen is sequentially dependent on IL-23 and IL-1beta. J Immunol. (2011) 187:532835. doi: 10.4049/jimmunol.1101445

35. Schroder K, Tschopp J. The inflammasomes. Cell (2010) 140:821-32. doi: 10.1016/j.cell.2010.01.040

36. Stutz A, Golenbock DT, Latz E. Inflammasomes: too big to miss. J Clin Invest. (2009) 119:3502-11. doi: 10.1172/JCI40599

37. Ritter M, Gross O, Kays S, Ruland J, Nimmerjahn F, Saijo S, et al. Schistosoma mansoni triggers Dectin-2, which activates the Nlrp3 inflammasome and alters adaptive immune responses. Proc Natl Acad Sci USA. (2010) 107:20459-64. doi: $10.1073 /$ pnas. 1010337107

38. Geijtenbeek TB, Gringhuis SI. C-type lectin receptors in the control of $\mathrm{T}$ helper cell differentiation. Nat Rev Immunol. (2016) 16:433-48. doi: $10.1038 /$ nri.2016.55

39. Ponichtera HE, Stadecker MJ. Dendritic cell expression of the C-type lectin receptor CD209a: a novel innate parasite-sensing mechanism inducing Th17 cells that drive severe immunopathology in murine schistosome infection. Exp Parasitol. (2015) 158:42-7. doi: 10.1016/j.exppara.2015.04.006

40. Kalantari P, Morales Y, Miller EA, Jaramillo LD, Ponichtera HE, Wuethrich MA, et al. CD209a synergizes with Dectin-2 and mincle to drive severe Th17 cell-mediated schistosome egg-induced immunopathology. Cell Rep. (2018) 22:1288-300. doi: 10.1016/j.celrep.2018.01.001

41. Koppel EA, van Gisbergen KP, Geijtenbeek TB, van Kooyk Y. Distinct functions of DC-SIGN and its homologues L-SIGN (DC-SIGNR) and 
mSIGNR1 in pathogen recognition and immune regulation. Cell Microbiol. (2005) 7:157-65. doi: 10.1111/j.1462-5822.2004.00480.x

42. Cheong C, Matos I, Choi JH, Dandamudi DB, Shrestha E, Longhi MP, et al. Microbial stimulation fully differentiates monocytes to DCSIGN/CD209(+) dendritic cells for immune T cell areas. Cell (2010) 143:41629. doi: 10.1016/j.cell.2010.09.039

43. den Dunnen J, Gringhuis SI, Geijtenbeek TB. Innate signaling by the C-type lectin DC-SIGN dictates immune responses. Cancer Immunol Immunother. (2009) 58:1149-57. doi: 10.1007/s00262-008-0615-1

44. Gringhuis SI, den Dunnen J, Litjens M, van der Vlist M, Geijtenbeek TB. Carbohydrate-specific signaling through the DC-SIGN signalosome tailors immunity to Mycobacterium tuberculosis, HIV-1 and Helicobacter pylori. Nat Immunol. (2009) 10:1081-8. doi: 10.1038/ni.1778

45. Gringhuis SI, Kaptein TM, Wevers BA, Mesman AW, Geijtenbeek TB. Fucose-specific DC-SIGN signalling directs $\mathrm{T}$ helper cell type-2 responses via IKKepsilon- and CYLD-dependent Bcl3 activation. Nat Commun. (2014) 5:3898. doi: $10.1038 /$ ncomms 4898

46. Geijtenbeek TB, den Dunnen J, Gringhuis SI. Pathogen recognition by DC-SIGN shapes adaptive immunity. Fut Microbiol. (2009) 4:879-90. doi: $10.2217 / \mathrm{fmb} .09 .51$

47. Zheng RB, Jegouzo SAF, Joe M, Bai Y, Tran HA, Shen K, et al. Insights into interactions of mycobacteria with the host innate immune system from a novel array of synthetic mycobacterial glycans. ACS Chem Biol. (2017) 12:2990-3002. doi: 10.1021/acschembio.7b00797

48. Meyer S, Tefsen B, Imberty A, Geyer R, van Die I. The C-type lectin LSIGN differentially recognizes glycan antigens on egg glycosphingolipids and soluble egg glycoproteins from Schistosoma mansoni. Glycobiology (2007) 17:1104-19. doi: 10.1093/glycob/cwm073

49. Takahara K, Yashima Y, Omatsu Y, Yoshida H, Kimura Y, Kang YS, et al. Functional comparison of the mouse DC-SIGN, SIGNR1, SIGNR3 and Langerin, C-type lectins. Int Immunol. (2004) 16:819-29. doi: 10.1093/intimm/dxh084

50. Powlesland AS, Ward EM, Sadhu SK, Guo Y, Taylor ME, Drickamer $\mathrm{K}$. Widely divergent biochemical properties of the complete set of mouse DC-SIGN-related proteins. J Biol Chem. (2006) 281:20440-9. doi: 10.1074/jbc.M601925200

51. Cummings RD, Nyame AK. Schistosome glysoconjugates. Biochim Biophys Acta (1999) 1455:363-74. doi: 10.1016/S0925-4439(99)00063-0

52. Hokke CH, Yazdanbakhsh M. Schistosome glycans and innate immunity. Parasite Immunol. (2005) 27:257-64. doi: 10.1111/j.1365-3024.2005.00781.x

53. Hokke $\mathrm{CH}$, Deelder AM. Schistosome glycoconjugates in host-parasite interplay. Glycoconj J. (2001) 18:573-87. doi: 10.1023/A:1020634602161

54. Prasanphanich NS, Mickum ML, Heimburg-Molinaro J, Cummings RD. Glycoconjugates in host-helminth interactions. Front Immunol. (2013) 4:240. doi: $10.3389 /$ fimmu.2013.00240
55. Saunders SP, Walsh CM, Barlow JL, Mangan NE, Taylor PR, McKenzie AN, et al. The C-type lectin SIGNR1 binds Schistosoma mansoni antigens in vitro, but SIGNR1-deficient mice have normal responses during schistosome infection. Infect Immun. (2009) 77:399-404. doi: 10.1128/IAI.00 762-08

56. van Liempt E, van Vliet SJ, Engering A, Garcia Vallejo JJ, Bank CM, SanchezHernandez M, et al. Schistosoma mansoni soluble egg antigens are internalized by human dendritic cells through multiple C-type lectins and suppress TLR-induced dendritic cell activation. Mol Immunol. (2007) 44:2605-15. doi: 10.1016/j.molimm.2006.12.012

57. Guo Y, Feinberg H, Conroy E, Mitchell DA, Alvarez R, Blixt O, et al. Structural basis for distinct ligand-binding and targeting properties of the receptors DC-SIGN and DC-SIGNR. Nat Struct Mol Biol. (2004) 11:591-8. doi: $10.1038 / \mathrm{nsmb} 784$

58. Gringhuis SI, den Dunnen J, Litjens M, van Het Hof B, van Kooyk Y, Geijtenbeek TB. C-type lectin DC-SIGN modulates Toll-like receptor signaling via Raf-1 kinase-dependent acetylation of transcription factor NF-kappaB. Immunity (2007) 26:605-16. doi: 10.1016/j.immuni.2007. 03.012

59. Sancho D, Reis e Sousa C. Signaling by myeloid C-type lectin receptors in immunity and homeostasis. Annu Rev Immunol. (2012) 30:491-529. doi: 10.1146/annurev-immunol-031210-101352

60. Hardison SE, Brown GD. C-type lectin receptors orchestrate antifungal immunity. Nat Immunol. (2012) 13:817-22. doi: 10.1038/ni.2369

61. Kerrigan AM, Brown GD. Syk-coupled C-type lectins in immunity. Trends Immunol. (2011) 32:151-6. doi: 10.1016/j.it.2011.01.002

62. Hou H, Guo Y, Chang Q, Luo T, Wu X, Zhao X. C-type lectin receptor: old friend and new player. Med Chem. (2017) 13:536-43. doi: 10.2174/1573406413666170510103030

63. Gringhuis SI, Wevers BA, Kaptein TM, van Capel TM, Theelen B, Boekhout $\mathrm{T}$, et al. Selective C-Rel activation via Malt1 controls anti-fungal $\mathrm{T}(\mathrm{H})$ 17 immunity by dectin-1 and dectin-2. PLoS Pathog. (2011) 7:e1001259. doi: 10.1371 /journal.ppat.1001259

Conflict of Interest Statement: The authors declare that the research was conducted in the absence of any commercial or financial relationships that could be construed as a potential conflict of interest.

Copyright $\odot 2019$ Kalantari, Bunnell and Stadecker. This is an open-access article distributed under the terms of the Creative Commons Attribution License (CC BY). The use, distribution or reproduction in other forums is permitted, provided the original author(s) and the copyright owner(s) are credited and that the original publication in this journal is cited, in accordance with accepted academic practice. No use, distribution or reproduction is permitted which does not comply with these terms. 